\title{
Reactant subspaces and kinetics of chemical reaction networks
}

\author{
Carlene Perpetua P. Arceo' ${ }^{1}$. Editha C. Jose ${ }^{2}$. \\ Angelyn R. Lao ${ }^{3,4}$. Eduardo R. Mendoza $2,3,5,6$
}

Received: 30 May 2017 / Accepted: 15 September 2017 / Published online: 5 October 2017 (C) The Author(s) 2017. This article is an open access publication

\begin{abstract}
This paper studies a chemical reaction network's (CRN) reactant subspace, i.e. the linear subspace generated by its reactant complexes, to elucidate its role in the system's kinetic behaviour. We introduce concepts such as reactant rank and reactant deficiency and compare them with their analogues currently used in chemical reaction network theory. We construct a classification of CRNs based on the type of intersection between the reactant subspace $R$ and the stoichiometric subspace $S$ and identify the subnetwork of $S$-complexes, i.e. complexes which, when viewed as vectors, are contained in $S$, as a tool to study the network classes, which play a key role in the kinetic behaviour. Our main results on new connections between reactant subspaces and kinetic properties are (1) determination of kinetic characteristics of CRNs with zero reactant deficiency by considering the difference between (network) deficiency and reactant deficiency, (2) resolution of the coincidence problem between the reactant and kinetic subspaces for complex factorizable kinetics via an analogue of the generalized Feinberg-Horn theorem, and (3) construction of an appropriate subspace for the parametrization and uniqueness of positive equilibria for complex factorizable power law kinetics, extending the work of Müller and Regensburger.
\end{abstract}

Eduardo R. Mendoza

mendoza@1mu.de

1 Institute of Mathematics, University of the Philippines Diliman, Quezon City 1101, Philippines

2 Institute of Mathematical Sciences and Physics, University of the Philippines Los Baños, Laguna 4031, Philippines

3 Mathematics and Statistics Department, De La Salle University, Manila 1004, Philippines

4 Mathematical and Statistical Modeling Research Unit, CENSER, De La Salle University, Manila 1004, Philippines

5 Max Planck Institute of Biochemistry, Martinsried, Munich, Germany

6 LMU Faculty of Physics, Geschwister -Scholl- Platz 1, 80539 Munich, Germany 
Keywords Chemical reaction networks - Power law kinetics - Reactant subspace ·

Reactant deficiency $\cdot S$-complexes $\cdot$ Kinetic-reactant subspace coincidence $\cdot$ Kinetic flux subspace

\section{List of symbols}

$\begin{array}{ll}I_{a}^{-} & \text {Branching map } \\ I_{a}^{+} & \text {Converse branching map } \\ \delta & \text { Deficiency of a CRN } \\ \psi_{K} & \text { Factor map of a complex factorizable kinetics } K \\ I_{a} & \text { Incidence map of a CRN } \\ \delta_{k} & k \text {-deficiency } \\ I_{k} & k \text {-incidence map } \\ A_{k} & k \text {-Laplacian map } \\ \widetilde{S} & \text { Kinetic flux space } \\ \widetilde{S} & \text { Kinetic rank } \\ \widetilde{q} & \text { Kinetic reactant rank } \\ R & \text { Kinetic reactant subspace } \\ K & \text { Kinetic subspace of a CKS } \\ Y & \text { Molecularity map/matrix of complexes } \\ \pi & \text { Product map } \\ P & \text { Product subspace } \\ \Delta(\mathscr{N}) & \text { Rank difference } \\ \rho & \text { Reactant map } \\ R & \text { Reactant subspace } \\ N & \text { Reactant subspace map } \\ S & \text { Stoichiometric subspace of a CRN } \\ \mathscr{N} S & \text { Subnetwork of } S \text {-complexes } \\ T & \text { T matrix }\end{array}$

\section{Abbreviations}

CFK Complex factorizable kinetics

CKS Chemical kinetic system

CRN Chemical reaction network

CRNT Chemical reaction network theory

FSK Factor span surjective kinetics

GMAK Generalized mass action kinetics

HRR High reactant rank

ILC Independent linkage classes

KSSC KSS coincidence

LRR Low reactant rank

MAK Mass action kinetics

MRR Medium reactant rank

NRS Nontrivial R-S intersection 


$\begin{array}{ll}\text { PLK } & \text { Power law kinetics } \\ \text { PL-RDK } & \text { Power law reactant-determined kinetics } \\ \text { PL-RLK } & \text { Power law reactant set linear independent kinetics } \\ \text { PL-YKA } & \text { Power law Y-kernel aligned kinetics } \\ \text { RES } & \text { Reactant subspace equal to stoichiometric subspace } \\ \text { RKS } & \text { Reactant-determined kinetic subspace } \\ \text { RSP } & S \text { proper subspace of } R \\ \text { RSS } & \text { Reactant-determined stoichiometric subspace } \\ \text { SCC } & \text { Stoichiometric compatibility class } \\ \text { SRP } & R \text { proper subspace of } S \\ \text { SRS } & R \text { subspace of } S \\ \text { TRS } & \text { Trivial R-S intersection }\end{array}$

\section{Introduction}

The linear subspace generated by a Chemical Reaction Network's (CRN) reactant complexes in species (composition) space $\mathbb{R}^{\mathscr{S}}$, which we call reactant subspace and denote by $R$, and its impact on a kinetic system's dynamics, have so far received little attention in Chemical Reaction Network Theory (CRNT). This is surprising in the sense that the coefficients of reactant complexes are important not only for the network's stoichiometry, but also, as the exponents of a mass action system's "monomial map", i.e. its "kinetic orders", determine the nonlinear behavior of the system. To our knowledge, the reactant subspace $R$ has been studied only in Injectivity Theory for mass action kinetics (MAK), which was pioneered by Craciun and Feinberg in a series of papers starting with [5]. In fact, one of the original motivations for our study was to understand a remark in [8] concerning a connection between the dimension of $R$ and the occurrence of non-degenerate equilibria.

The second motivation for our study of reactant subspaces stems from our analysis of BST systems in [2], which highlighted the prevalent occurrence of terminal points in their CRN representations. Since the number of terminal points in a CRN is given by the difference $n-n_{r}$ ( $n=$ number of complexes and $n_{r}=$ number of reactant complexes), this led to the realization of the importance of the invariant $n_{r}$ in such networks. Deficiency theory in CRNT has mainly focused on weakly reversible networks, which form a subset of CRNs, in which each complex is a reactant complex, i.e. $n=n_{r}$ (which we call cycle terminal networks), and hence has not considered the invariant and related properties. For example, to date, there is no CRNT software tool that automatically calculates $n_{r}$ for a given network. Our considerations led us eventually to study a superset of cycle terminal and terminal point containing networks with the property that the stoichiometric subspace $S \subset R$ (which we call $R S S$ $=$ reactant-determined stoichiometric subspace), which possesses many interesting kinetic properties, in particular, with respect to power law kinetics.

The study of RSS networks evolved to a full CRN classification based on the type of the intersection $R \cap S$. We demonstrate the key role of these network classes in two significant problems concerning kinetics in the latter part of the paper.

We have structured the paper as follows: 
In Sect. 2, we collect the fundamentals of chemical reaction networks (CRNs) and kinetics required for this Introduction and the latter sections. We expound the viewpoint that CRNs are digraphs with a vertex-labelling called "stoichiometry". This approach allows easier application of general digraph theory to CRNT, and vice versa, easier appreciation of original results from CRNT of relevance in general digraph theory.

In Sect. 3, we begin with the systematic study of the reactant subspace, highlighting similarities to and differences with the stoichiometric subspace. We introduce concepts such as reactant rank and rank difference, which play an important role in connection to kinetics. The main result in this section (Theorem 1) is a formula for the difference between (network) deficiency and reactant deficiency, which we use to determine existence and characteristics of positive equilibria of kinetics on CRNs with zero reactant deficiency. A brief introduction to the product subspace, the image of the reactant subspace under the converse digraph transformation, concludes the section.

In Sect. 4, we deepen our study of the reactant subspace by introducing a classification of CRNs based on the intersection $R \cap S$. The network classes play an important role in the reactant subspace's connection to kinetic behavior. The main result in this section (Theorem 2) characterizes the network classes in terms of the containment of $R$ and $S$ in $\operatorname{Im} Y$ and the subnetwork of $S$-complexes, a new tool that we introduce.

In Sect. 5, we discuss coincidence problems of the kinetic subspace $K$, first studied by Feinberg and Horn for $K$ and the stoichiometric subspace $S$ of MAK systems in 1977. The Feinberg-Horn KSSC (Kinetic and Stoichiometric Subspace Coincidence) Theorem was extended to complex factorizable kinetics in [2]. The main result in this section (Theorem 5) derives an analogue, the KRSC (Kinetic and Reactant Subspace Coincidence) Theorem for complex factorizable kinetics. The striking fact that $K$ and $R$ coincidence can occur only in the network class with $R \cap S=R$ follows from these considerations.

In Sect. 6, we discuss the problem of constructing a kinetic analogue of the stoichiometric subspace. Such a space is important for parametrization and uniqueness questions of positive equilibria of complex factorizable power law kinetic systems (denoted PL-RDK), as shown by previous work of Müller and Regensburger on cycle terminal networks [13]. The main result in the section (Theorem 6) constructs such a "kinetic flux subspace" satisfying the requirements of coincidence with the stoichiometric subspace for MAK systems and with the kinetic order subspace of Müller-Regensburger for PL-RDK systems on cycle terminal networks. A key observation is that these requirements imply that the construction can occur only when $R \cap S=S$.

\section{Fundamentals of chemical reaction networks and kinetic systems}

In this section, we briefly go through the fundamental concepts of CRNs and chemical kinetic systems (CKS) needed for our results. We start by expounding the standpoint that a CRN is a digraph with a vertex-labelling. In our view, this approach not only allows us to apply (general) digraph theory results to CRNT, it also produces novel contributions to the (general) digraph theory. We focus on the CKS side of complex factorizable (CFK) and power-law (PLK) kinetic systems. 


\subsection{Chemical reaction networks as vertex-labelled digraphs}

Definition 1 A chemical reaction network is a digraph $D=(\mathscr{C}, \mathscr{R})$ where each vertex has positive degree and stoichiometry, i.e. there is a finite set $\mathscr{S}$ (whose elements are called species) such that $\mathscr{C}$ is a subset of $\mathbb{Z}_{\geq}^{\mathscr{S}}$. Each vertex is called a complex and its coordinates in $\mathbb{Z}_{\geq}^{\mathscr{S}}$ are called stoichiometric coefficients. The arcs are called reactions. We denote the number of species with $m$, the number of complexes with $n$ and the number of reactions with $r$.

Stoichiometry is a special property of CRN. It can be viewed as a vertex-labelling and allows the embedding of the graph's vertices in the real vector space $\mathbb{R}^{\mathscr{S}}$, called species composition space (or simply species space). Its elements are (chemical) compositions such that the coordinate values are concentrations of the different (chemical) species. The standard notation to specify a $\mathrm{CRN}$ as a triple $(\mathscr{S}, \mathscr{C}, \mathscr{R})$ is thus indicated as the pair $((\mathscr{C}, \mathscr{R}), \mathscr{S})$.

Moreover, if $\mathscr{S}=\left\{X_{1}, \ldots, X_{m}\right\}$ then $X_{i}$ can be identified with the vector with 1 in the $i^{\text {th }}$ coordinate and zero otherwise. As such that $\mathscr{S}=\cup \operatorname{supp} y$, for $y \in \mathscr{C}$, each species should appear in at least one of the complexes.

A complex is called monospecies if it consists of only one species, i.e. of the form $n X_{i}, n$ a non-negative integer and $X_{i}$ a species. It is called monomolecular if $n=1$, and is identified with the zero complex for $n=0$. Zero complex is a special property of CRNs. It represents the "outside" of the system studied, from which chemicals can flow into the system at a constant rate and to which they can flow out at a linear rate (proportional to the concentration of the species). In biological systems, the "outside" also stands for the degradation of a species. An inflow reaction is a reaction with source " 0 " and an outflow reaction is a reaction with a monomolecular complex as source and the zero complex as target, respectively.

Below we define some useful maps that are associated with each reaction:

Definition 2 The reactant map $\rho: \mathscr{R} \rightarrow \mathscr{C}$ maps a reaction to its reactant complex while the product map $\pi: \mathscr{R} \rightarrow \mathscr{C}$ maps it to its product complex. We denote $|\rho(\mathscr{R})|$ with $n_{r}$, i.e. the number of reactant complexes. The reactions map $\rho^{\prime}: \mathbb{R}^{\mathscr{C}} \rightarrow \mathbb{R}^{\mathscr{R}}$ maps $f: \mathscr{C} \rightarrow \mathbb{R}$ to $f \circ \rho$, i.e., $\rho^{\prime}(f)=f \circ \rho$.

The reactant map of a CRN is surjective iff $n_{r}=n$. In this case, the CRN is cycle terminal. On the other hand, the reactant mapping $\rho$ of a CRN is injective iff $n_{r}=r$, which gives a nonbranching CRN.

\subsection{Stoichiometry-independent properties of CRNs}

\subsubsection{Connectivity in a CRN}

Connectivity in a digraph that is applied to CRNs is traditionally called a linkage class in CRNT. A subset of this linkage class where any two elements are connected by a directed path in each direction is known as the strong linkage class. If there is no reaction from a complex in the strong linkage class to a complex outside the same 
strong linkage class, then we have a terminal strong linkage class. We denote the number of linkage classes with $l$, that of the strong linkage classes with $s l$, and that of terminal strong linkage classes with $t$. Clearly $s l \geq t \geq l$. A chemical reaction network is called weakly reversible if $s l=l$. It is called $t$-minimal if $t=l$.

For each linkage class $\mathscr{L}_{i}$ that forms a subnetwork, its number of complexes and reactions in $\mathscr{L}_{i}$ are denoted by $n_{i}$ and $r_{i}$ respectively, $i=1,2, \ldots, l$. With $\mathscr{C}^{i}$ as the set of complexes in linkage class $\mathscr{L}_{i}$, we set $e^{1}, e^{2}, \ldots, e^{l} \in\{0,1\}^{n}$ as the characteristic vectors of the sets $\mathscr{C}^{1}, \mathscr{C}^{2}, \ldots, \mathscr{C}^{l}$, respectively.

There are two types of terminal (strong linkage) classes in a CRN: cycles (not necessarily simple) and singletons (which we call "terminal points"). If $t_{c}=$ number of cycle terminal classes and $t_{p}=$ number of point terminal classes, then $t=t_{c}+t_{p}$. Note also that $n-n_{r}=t_{p}=t-t_{c}$. A CRN is cycle terminal if $t_{p}=0$ (i.e. $n=n_{r}$ ), point terminal if $t_{c}=0$ (i.e. $t=n-n_{r}$ ) and point and cycle terminal otherwise (i.e. $t_{p}>0$ and $t_{c}>0$ or equivalently, $t>n-n_{r}$ ).

\subsubsection{Linear algebra of a $C R N$}

Here, we start by introducing a fundamental invariant of a digraph, that is the incidence map $I_{a}: \mathbb{R}^{\mathscr{R}} \rightarrow \mathbb{R}^{\mathscr{C}}$. With $f: \mathscr{R} \rightarrow \mathbb{R}$, it is defined as $I_{a}(f)(v)=-f(a)$ and $f(a)$ if $v=\rho(a)$ and $v=\pi(a)$, respectively, and are 0 otherwise. Equivalently, it maps the basis vector $\omega_{a}$ to $\omega_{v^{\prime}}-\omega_{v}$ if $a: v \rightarrow v^{\prime}$. It is clearly a linear map, and its matrix representation (with respect to the standard bases $\omega_{a}, \omega_{v}$ ) is called the incidence matrix and can be described as

$$
\left(I_{a}\right)_{i, j}= \begin{cases}-1 & \text { if } \rho\left(a_{j}\right)=v_{i} \\ 1 & \text { if } \pi\left(a_{j}\right)=v_{i} \\ 0 & \text { otherwise }\end{cases}
$$

Note that in most digraph theory books, the incidence matrix is set as $-I_{a}$.

An important result of digraph theory regarding the incidence matrix is the following:

Proposition 1 Let I be the incidence matrix of the directed graph $D=(V, E)$. Then rank $I=n-l$, where $l$ is the number of connected components of $D$.

Besides the vertex-labelling via stoichiometry, arc labels are often associated with a CRN, i.e. a map $k: \mathscr{R} \rightarrow \mathbb{R}_{>}$is specified. Several linear maps are associated with such $k$-labelled CRNS:

Definition 3 The $k$-diagonal map diag $(k)$ maps $\omega_{r}$ to $k_{r} \omega_{r}$. The $k$-incidence map $I_{k}$ is defined as the composition diag $(k) \circ \rho^{\prime}$. The $k$-Laplacian map $A_{k}: \mathbb{R}^{\mathscr{C}} \rightarrow \mathbb{R}^{\mathscr{C}}$ is defined as the composition $A_{k}=I_{a} \circ I_{k}$.

The $k$-diagonal map is clearly a linear isomorphism and maps the positive orthant $\mathbb{R}_{>}^{\mathscr{R}}$ onto itself. In fact, as pointed out in [11], all such maps are $k$-diagonal maps:

Proposition 2 ([11]) A linear, bijective mapping $h: \mathbb{R}_{>}^{\mathscr{R}} \mapsto \mathbb{R}_{>}^{\mathscr{R}}$ may consist of at most positive scaling and reindexing of coordinates. 
Proposition 3 ([2]) For any $k$-incidence map, dim ker $I_{k}=n-n_{r}$ and $\operatorname{dim} \operatorname{Im} I_{k}=$ $n_{r}$. The mapping $I_{k}$ is injective iff $\mathscr{N}$ is cycle terminal and surjective iff $\mathscr{N}$ is nonbranching.

\subsection{Stoichiometry-dependent properties of a CRN}

\subsubsection{The stoichiometric map and matrix}

The properties of stoichiometry and embedding in composition space add two important maps to the linear algebra view: the map of complexes $Y$ and the stoichiometric map $N$, which we define in the following.

Definition 4 The map of complexes $Y: \mathbb{R}^{\mathscr{C}} \rightarrow \mathbb{R}^{\mathscr{S}}$ is defined by its values on the standard basis $\left\{\omega_{y}\right\}$ where $y$ is a complex. It is given by $Y\left(\omega_{y}\right)=y$ and extends linearly to all elements of $\mathbb{R}^{\mathscr{C}}$. Clearly, its matrix (matrix of complexes; also denoted with $Y$ ) is an $m \times n$ matrix, its rows indexed by the species and its columns by the complexes, with $y_{i j}$ being the stoichiometric coefficient of the $j$ th complex in the $i$ th species. In other words, the columns are the complexes written as column vectors.

Remark 1 One often leaves out the column of zeros (for the zero complex) when specifying the matrix $Y$.

Definition 5 The stoichiometric map $N: \mathbb{R}^{\mathscr{R}} \rightarrow \mathbb{R}^{\mathscr{S}}$ is defined as $N=Y \circ I_{a}$. $N$ also denotes its matrix (called the stoichiometric matrix), whose elements are the differences of the stoichiometric coefficients of the product complex (target) and the reactant (source) complex per species.

The kernel of the stoichiometric map $(\operatorname{ker} N)$ contains $\operatorname{ker} I_{a}$ and plays a central role in flux-oriented analysis (also summarily called "Stoichiometric Analysis") in Systems Biology. It is called the nullspace of the CRN.

\subsubsection{The stoichiometric subspace of a $C R N$}

Further examples of the "linear algebraic" view are given by the following definitions and proposition.

Given the reaction vectors for a reaction network $(\mathscr{S}, \mathscr{C}, \mathscr{R})$ that are the members of the set $\left\{y^{\prime}-y \in \mathbb{R}^{\mathscr{S}}: y \rightarrow y^{\prime} \in \mathscr{R}\right\}$, the stoichiometric subspace $S$ of a reaction network $(\mathscr{S}, \mathscr{C}, \mathscr{R})$ is the linear subspace of $\mathbb{R}^{\mathscr{S}}$ defined by

$$
S:=\operatorname{span}\left\{y^{\prime}-y \in \mathbb{R}^{\mathscr{S}}: y \rightarrow y^{\prime} \in \mathscr{R}\right\}=\operatorname{Im} N \subset \operatorname{Im} Y .
$$

The rank of a CRN, $s$, is defined as $s=\operatorname{dim} S$.

The next proposition clarifies the relationship between $S$ and $N$.

Proposition $4 S=\operatorname{Im}(N)$ and $\operatorname{dim}(\operatorname{ker} N)=r-s$. 
The concepts of stoichiometric subspace and rank can be applied to the linkage classes of a CRN.

Definition 6 In a reaction network $(\mathscr{S}, \mathscr{C}, \mathscr{R})$, the rank of linkage class $\mathscr{L}$, denoted by $s^{\mathscr{L}}$, is the rank of the set

$$
\left\{y^{\prime}-y \in \mathbb{R}^{\mathscr{S}} \mid y \rightarrow y^{\prime}, y \in \mathscr{L}, y^{\prime} \in \mathscr{L}\right\}
$$

Through elementary algebraic considerations, it follows that the rank of the network and the ranks of the linkage classes must be related in the following way:

$$
s \leqq \sum_{\mathscr{C} / \sim} s^{\mathscr{L}} .
$$

\subsubsection{The stoichiometric (compatibility) classes of a CRN}

Definition 7 Two elements $a, b$ of $\mathbb{R}^{\mathscr{S}}$ are stoichiometrically compatible if $a-b$ is contained in $S$. The intersection of a coset $c+S$ with $\mathbb{R}_{\geq}^{\mathscr{S}}$ is called a stoichiometric compatibility class.

We note that stoichiometric compatibility is an equivalence relation that partitions $\mathbb{R}_{\geq}^{\mathscr{S}}$ into equivalence classes. Thus, the stoichiometric compatibility class (SCC) containing an arbitrary composition $c$, denoted $(c+S) \cap \mathbb{R}_{\geq}^{\mathscr{S}}$, is given by

$$
(c+S) \cap \mathbb{R}_{\geq}^{\mathscr{S}}=\left\{c^{\prime} \in \mathbb{R}_{\geq}^{\mathscr{S}}: c^{\prime}-c \in S\right\}
$$

In recent papers, various authors use the shorter term "stoichiometric class" for an SCC. A stoichiometric class is also called an invariant polyhedron of the CRN.

A stoichiometric compatibility class will typically contain a wealth of (strictly) positive compositions. We say that a stoichiometric compatibility class is nontrivial if it contains a member of $\left(\mathbb{R}_{>}\right)^{\mathscr{S}}$. To see that a stoichiometric compatibility class can be trivial, consider the simple reaction network $A+B \leftrightarrows C$, and let $\bar{c}$ be the composition defined by $\bar{c}_{A}=1, \bar{c}_{B}=0, \bar{c}_{C}=0$. Then the stoichiometric compatibility class containing $\bar{c}$ has $\bar{c}$ as its only member. Such a network is called open. If the stoichiometric subspace $S=\mathbb{R}^{\mathscr{S}}$, then there is only one SCC. A fully open network is an example for this condition.

\subsection{The deficiency concepts of a CRN}

A central concept of the theory of chemical reaction networks is the deficiency of the $\mathrm{CRN}$, defined as $\delta=n-l-s$. Geometrically, it is interpreted as $\operatorname{dim}\left(\operatorname{ker} Y \cap \operatorname{Im} I_{a}\right)$. The deficiency measures the amount of linear independence among the reactions of the network. The higher the deficiency, the lower the extent of linear independence [15]. 
Definition 8 The deficiency of linkage class $\mathscr{L}$ (denoted by $\delta^{\mathscr{L}}$ ) is defined by the formula

$$
\delta^{\mathscr{L}}:=n^{\mathscr{L}}-1-s^{\mathscr{L}}
$$

where $n^{\mathscr{L}}$ is the number of complexes in linkage class $\mathscr{L}$.

From the preceding definition and the fact that $\mathscr{C}$ is the disjoint union of the linkage classes, it follows that the deficiency of the network and the deficiencies of its linkage classes must satisfy the relation

$$
\delta \geqq \sum_{\mathscr{C} / \sim} \delta^{\mathscr{L}} .
$$

Moreover, inequality holds in Eq. 3 if and only if inequality holds in Eq. 1.

Some authors have defined $k$-deficiency as a deficiency of a network (e.g. Gunawardena [10], Otero-Murras et al. [12,14]) because they mainly considered weakly reversible networks where the two values coincide (see below). Following our "structural view", we associate it with a positive vector $k$.

Definition 9 The $k$-deficiency function of a CRN def $: \mathbb{R}_{>}^{\mathscr{R}} \rightarrow \mathbb{N}_{0}$ assigns to each vector $k$ the non-negative integer $\delta_{k}=\operatorname{dim}\left(\operatorname{ker} Y \cap \operatorname{Im} A_{k}\right)$.

Since $\operatorname{Im} A_{k} \subset \operatorname{Im} I_{a}$, we have $\operatorname{ker} Y \cap \operatorname{Im} A_{k} \subset \operatorname{ker} Y \cap \operatorname{Im} I_{a} \Rightarrow \delta_{k} \leq \delta$, so that the function is bounded.

The following Proposition provides more specific bounds for the function:

Proposition 5 ([2]) $\delta_{k} \geq \delta+l-t$. Moreover, Im $Y A_{k}=S$ iff equality holds.

The upper and lower bounds for the $k$-deficiency function can be formulated as

$$
n-s-l \geq \delta_{k} \geq n-s-t,
$$

with the following special cases:

1. If $t-l=0$, then it follows that $\delta_{k}=\delta$ (again) and $\operatorname{Im} Y A_{k}=S$ for all $k$.

2. If $t-l>\delta$, then $0>\delta+l-t$, hence inequality holds and $\operatorname{Im} Y A_{k} \neq S$ for all $k$.

3. If $0<t-l \leq \delta, \operatorname{Im} Y A_{k}=S$ or $\operatorname{Im} Y A_{k} \neq S$ depends on the choice of $k$.

\subsection{Fundamentals of chemical kinetic systems}

In [2], we introduced a slightly more general definition of a kinetics. We say a kinetics for a network $\mathscr{N}=(\mathscr{S}, \mathscr{C}, \mathscr{R})$ is an assignment to each reaction $r_{j} \in \mathscr{R}$ of a rate function $K_{j}: \Omega_{K} \rightarrow \mathbb{R}_{\geq}$, where $\Omega_{K}$ is a set such that $\mathbb{R}_{>}^{\mathscr{S}} \subseteq \Omega_{K} \subseteq \mathbb{R}_{\geq}^{\mathscr{S}}, c \wedge d \in \Omega_{K}$ whenever $c, d \in \Omega_{K}$, and

$$
K_{j}(c) \geq 0, \forall c \in \Omega_{K}
$$

A kinetics for a network $\mathscr{N}$ is denoted by $K=\left(K_{1}, K_{2}, \ldots, K_{r}\right): \Omega_{K} \rightarrow \mathbb{R}_{\geq}^{\mathscr{R}}$.

We focus on its subset relevant to our context: 
Definition 10 A chemical kinetics is a kinetics $K$ satisfying the positivity condition: for each reaction $j: y \rightarrow y^{\prime}, K_{j}(c)>0$ iff supp $y \subset \operatorname{supp} c$.

The species formation rate function (SFRF) of a chemical kinetic system (CKS) is the vector field

$$
f(x)=N K(x)=\sum_{y \rightarrow y^{\prime}} K_{y \rightarrow y^{\prime}}(x)\left(y^{\prime}-y\right) .
$$

The equation $d x / d t=f(x)$ is the ODE or dynamical system of the CKS. A zero of $f$ is an element $c$ of $\mathbb{R}^{\mathscr{S}}$ such that $f(c)=0$. A zero of $f$ is an equilibrium or steady state of the ODE system. For a differentiable $f$, a steady state $c$ is called non-degenerate if $\operatorname{ker}\left(J_{c}(f)\right) \cap S=\{0\}$, where $J_{c}(f)$ is the Jacobian matrix of $f$ at $c$.

The difference between production and degradation for each complex is called the "complex formation rate function" and given by the function $g=I_{a} K: \mathbb{R}^{\mathscr{S}} \rightarrow \mathbb{R}^{\mathscr{C}}$.

A fundamental connection between stoichiometry and kinetics is given by the fact that the trajectory of the chemical system is contained in the stoichiometric compability class of its initial point ([6], Lemma 2.2). Hence, all questions relating to existence and number of steady states are relative to a stoichiometric compatibility class. In particular, a chemical kinetic system is multistationary (or has the capability for multiple steady states) if there is at least one stoichiometric compatibility class with two distinct steady states. Conversely, it is monostationary, if for all stoichiometric compatibility classes, it has at most one steady state.

\subsection{Span surjectivity in kinetic systems}

We recall from [2] that a mapping $w: \mathbb{R}^{u} \rightarrow \mathbb{R}^{v}$ is called span surjective if $\operatorname{span}(\operatorname{Im} w)=\mathbb{R}^{v}$. It was shown in [2] that $w$ is span surjective iff its component functions $w_{1}, \ldots, w_{v}$ are linearly independent over $\mathbb{R}$.

A kinetics can be viewed as a mapping from $\mathbb{R}^{\mathscr{S}}$ to $\mathbb{R}^{\mathscr{R}}$. If it is span surjective then it is called a span surjective kinetics. Span surjectivity induces a nice relationship between the kinetic space $K$ and stoichiometric subspace $S$ of a CRN.

Proposition 6 ([2]) If a chemical kinetics $K: \mathbb{R}^{\mathscr{S}} \rightarrow \mathbb{R}^{\mathscr{R}}$ is span surjective, then $K=S$.

Proposition 7 ([2]) A PLK system is span surjective iff all rows in the kinetic order matrix $F$ are pairwise different (i.e. $r \neq r^{\prime} \Rightarrow F_{r} \neq F_{r^{\prime}}$ ).

The preceding result proved in [2] used a generalization of a well-known fact that if $f_{i}=\prod X_{j}^{g_{i j}}$, with $g_{i}=\left(g_{i 1}, \ldots, g_{i n}\right)$ in $\mathbb{R}^{n}$ then $f_{1}, f_{2}, \ldots, f_{m}$ are linearly independent (over $\mathbb{R})$ iff the $g_{i}$ are pairwise different. 


\subsection{Complex factorizable kinetics}

We refine the definition of a complex factorizable kinetics (CFK) to accommodate a more general domain $\Omega_{K}$ and a more appropriate codomain:

Definition 11 A chemical kinetics $K: \Omega_{K} \rightarrow \mathbb{R}_{\geq}^{\mathscr{R}}$ is complex factorizable if there is a $k \in \mathbb{R}_{>}^{\mathscr{R}}$ and a mapping $\Psi_{K}: \Omega_{K} \rightarrow \mathbb{R}^{\rho(\mathscr{R})}$ such that $K=I_{k} \circ \Psi_{K}$. The set of complex factorizable kinetics is denoted as $\mathscr{C} \mathscr{F} \mathscr{K}(\mathscr{N})$.

It can be deduced from the definition that if a chemical kinetics $K$ is complex factorizable, then its complex formation rate function $g=A_{k} \circ \Psi_{K}$ and its species formation rate function $f=Y \circ A_{k} \circ \Psi_{K}$.

In [3] (see also [2]), we introduce a special subset of $\mathscr{C} \mathscr{F} \mathscr{K}(\mathscr{N})$, which is the set of power law kinetics with reactant-determined kinetic orders, denoted by $\mathscr{P} \mathscr{L}-$ $\mathscr{R} \mathscr{D} \mathscr{K}(\mathscr{N})$. A PLK system has reactant-determined kinetic orders (of type PLRDK) if for any two reactions $i, j$ with identical reactant complexes, the corresponding rows of kinetic orders in $V$ are identical, i.e., $v_{i k}=v_{j k}$ for $k=1,2, \ldots, m$.

We note also in [2] that $\mathscr{P} \mathscr{L}-\mathscr{R} \mathscr{D} \mathscr{K}(\mathscr{N})$ includes mass action kinetics (MAK) and coincides with the set of GMAK systems recently introduced by Müller and Regensburger [13]. They also constitute the subset of power law systems for which various authors claimed that their results "hold for complexes with real coefficients" are valid.

Another important property of a complex factorizable kinetics is "factor span surjectivity":

Definition 12 A complex factorizable kinetics $K$ is factor span surjective if its factor map $\Psi_{K}$ is span surjective. $\mathscr{F} \mathscr{S} \mathscr{K}(\mathscr{N})$ denotes the set of factor span surjective kinetics on a network $\mathscr{N}$.

We characterized in [2] a factor span surjective PL-RDK system.

Proposition 8 A PL-RDK system is factor span surjective iff all rows with different reactant complexes in the kinetic order matrix $F$ are pairwise different (i.e. $\rho(r) \neq$ $\left.\rho\left(r^{\prime}\right) \Rightarrow F_{r} \neq F_{r^{\prime}}\right)$.

\section{The reactant subspace of a CRN and related structures}

In this section, we begin our systematic study of the reactant subspace $R$, i.e. the linear space generated by the reactant complexes. We show that, as with the stoichiometric subspace, it is the image of a linear map $\mathbb{R}^{\mathscr{R}} \rightarrow \mathbb{R}^{\mathscr{S}}$, and introduce analogous concepts such as reactant rank and reactant deficiency. Our main result in this section shows that the difference between (network) deficiency and reactant deficiency is determined by the network's terminal class structure and its rank difference. In particular, cycle terminal networks with zero reactant deficiency also have zero (network) deficieny, while those with terminal points may have positive deficiency. 


\subsection{Basic properties of the reactant subspace}

Definition 13 The reactant subspace $R$ is the linear space in $\mathbb{R}^{\mathscr{S}}$ generated by the reactant complexes, i.e. $\langle\rho(\mathscr{R})>$. The reactant rank of the network is denoted by $q:=\operatorname{dim} R$.

We denote $\operatorname{dim} R$ with " $q$ " (since " $r$ " is already reserved for the number of reactions). We also denote $\operatorname{dim} \operatorname{Im} Y$ with “ $c$ " (since $\operatorname{Im} Y$ consists precisely of the network's complexes embedded in $\mathbb{R}^{\mathscr{S}}$ ).

We first note that since every reactant complex $y$ is the image of $\omega_{y}$ under $Y$, then $R \subset \operatorname{Im} Y$, just as $S \subset Y$. We further broaden the analogy between $R$ and $S$ by showing that $R$ too is the image of a linear map $N^{-}: \mathbb{R}^{\mathscr{R}} \rightarrow \mathbb{R}^{\mathscr{S}}$. To construct the map, we first introduce the branching map: if one wrote the incidence matrix $I_{a}=I_{a}^{+}-I_{a}^{-}$, where the first term consisted only of the 1's and 0's in $I_{a}$ and the second with only the zeros and the absolute values of the -1 's, the latter provides a precise way to identify the source vertices together with their branching behaviour (i.e. the number of branching reactions is the number of 1's in a row).

Definition 14 The linear map $I_{a}^{-}: \mathbb{R}^{\mathscr{R}} \rightarrow \mathbb{R}^{\mathscr{C}}$ defined by the matrix $I_{a}^{-}$is called the branching map of the CRN.

We have the following proposition:

Proposition 9 The image of the branching map is $\mathbb{R}^{\rho(\mathscr{R})}$, hence, its dimension equals $n_{r}$. Its kernel is trivial iff the CRN is nonbranching.

Proof A basis of $\mathbb{R}^{\rho(R)}$ is given by $-\omega_{y}$, where $y$ is a reactant complex, hence there must be at least one reaction mapped to it. The dimension of the kernel is $r-n_{r}$, from which the second claim follows.

We can now define $N^{-}$and determine its image:

Definition 15 The reactant subspace map $N^{-}: \mathbb{R}^{\mathscr{R}} \rightarrow \mathbb{R}^{\mathscr{S}}$ is defined as $N^{-}=$ $Y \circ I_{a}^{-}$.

Proposition 10 For any $C R N, \operatorname{Im} N^{-}=R$.

Proof $\operatorname{Im} N^{-}=Y\left(I_{a}^{-}\left(\mathbb{R}^{\mathscr{R}}\right)=Y\left(\mathbb{R}^{\rho(\mathscr{R})}\right)\right.$ since $I_{a}^{-}$is surjective, which is equal to $Y_{\text {res }}\left(\mathbb{R}^{\rho(\mathscr{R})}\right)=R$, where $Y_{\text {res }}$ is the restriction of $Y$ to $\mathbb{R}^{\rho(\mathscr{R})}$.

Remark 2 The previous proposition justifies the name for $N^{-}$. The dimension of its kernel equals $r-q$ (again in analogy to $\operatorname{dim} \operatorname{ker} N=r-s$ ). This analogy also justifies our calling $\operatorname{dim} R$ the reactant rank of the network.

The relationship of the reactant rank to the network's rank is important in the study of the reactant subspace and we introduce some relevant concepts:

Definition 16 The rank difference $\Delta(\mathscr{N})$ is equal to $s-q$. The network has high reactant rank (HRR) if $\Delta(\mathscr{N})$ is negative, medium reactant rank (MRR) if it is zero and low reactant rank (LRR) if it is positive.

We will discuss the relationship between $q$ and $s$, especially the rank difference, in more detail in the next two sections. In addition to $q \leq c \leq m$ from the above considerations, since there are $n_{r}$ reactant complexes, $q \leq n_{r}$. 


\subsection{Deficiency and reactant deficiency}

We introduce the essential measure of the linear independence of reactant complexes:

Definition 17 The reactant deficiency of a network is given by $\delta_{\rho}:=n_{r}-q$.

We also have shown the geometric interpretation of reactant deficiency:

$$
\delta_{\rho}=\operatorname{dim}\left(\operatorname{ker} Y \cap \operatorname{Im} I_{a}^{-}\right) .
$$

Remark 3 We use the subscript " $\rho$ " since we use the same symbol for the reactant map and also already introduced the subscript " $R$ " for the regulatory deficiency of a BST representation.

Remark 4 If a $\mathrm{CRN}$ has an inflow reaction, then its reactant deficiency is greater than 0 .

A natural question is: what is the relationship between reactant deficiency and (network) deficiency? To answer this question, we first introduce further concepts regarding the terminal class structure of a CRN.

M. Feinberg and F. Horn were the first to study the (non-negative) integer $t-l$, which played a crucial role in their solution of the kinetic and stoichiometric subspace coincidence problem for MAK systems (see Sect. 5 for details). As it also plays a significant role in our considerations, we give it a formal name:

Definition 18 The terminality of a CRN is the non-negative integer $\tau(\mathscr{N}):=t-l$.

In this terminology, a $\mathrm{CRN} \mathscr{N}$ is t-minimal iff $\tau(\mathscr{N})=0$.

Our main result in this section shows that the difference between deficiency and reactant deficiency is determined by the CRN's terminal class structure (a stoichiometry-independent term) and its rank difference (a stoichiometry-depedent one):

Theorem 1 Let $\mathscr{N}$ be a CRN with network deficiency $\delta$ and reactant deficiency $\delta_{\rho}$. Then

$$
\delta-\delta_{\rho}=\tau(\mathscr{N})-t_{c}-\Delta(\mathscr{N})
$$

In particular,

(i) if $\mathscr{N}$ is cycle terminal, then $0 \leq \delta_{\rho}-\delta=l+\Delta(\mathscr{N}) \leq l$;

(ii) if $\mathscr{N}$ is point terminal, then $\delta-\delta_{\rho}=\tau(\mathscr{N})-\Delta(\mathscr{N})$;

(iii) if $\mathscr{N}$ is point and cycle terminal, then $\delta-\delta_{\rho}<\tau(\mathscr{N})-\Delta(\mathscr{N})$.

Proof $\delta-\delta_{\rho}=n-l-s-n_{r}+q=n-n_{r}-l-s+q=\tau(\mathscr{N})-t_{c}-\Delta(\mathscr{N})$.

(i) If $\mathscr{N}$ is cycle terminal, $t_{p}=n-n_{r}=0 \Leftrightarrow t=t_{c} \Leftrightarrow \tau(\mathscr{N})-t_{c}=-l$. Hence, $\delta-\delta_{\rho}=-l-\Delta(\mathscr{N})$. Since $R=\operatorname{Im} Y, q=c \geq s$, and $\Delta(\mathscr{N})$ is negative. Hence $\delta_{\rho}-\delta=l+\Delta(\mathscr{N}) \leq l$. For the lower bound: $\delta_{\rho}=n_{r}-q=n-q \geq$ $n-c=\operatorname{dim} \operatorname{ker} Y \geq \operatorname{dim}\left(\operatorname{ker} Y \cap \operatorname{Im} I_{a}\right)=\delta$. 
(ii) If $\mathscr{N}$ is point terminal, $t_{c}=0$, hence the simpler formula.

(iii) If $\mathscr{N}$ is point and cycle terminal, then $t_{c}>0$, which implies the inequality.

\subsection{Kinetics on zero reactant deficiency CRNs}

In this section, we use Theorem 1 to derive properties of kinetics on zero reactant deficiency CRNs.

We have two immediate corollaries for cycle terminal networks:

Corollary 1 Any cycle terminal CRN with zero reactant deficiency also has network deficiency equal to 0 .

Corollary 2 If a cycle terminal network has the ILC property (i.e. $\delta_{1}+\delta_{2}+\cdots+\delta_{l}=$ $\delta)$, then $\sum \delta_{\rho, i}=0$ implies $\delta=0$.

Proof $0=\sum \delta_{\rho, i} \geq \sum \delta_{i}=\delta$.

Weakly reversible networks form an important subset of cycle terminal networks. Hence, a weakly reversible network with $\delta_{\rho}=0$ also has $\delta=0$. For a weakly reversible network with $\delta=0$, it follows from the Deficiency Zero Theorem (DZT) for MAK systems that it has a unique equilibrium in any stoichiometric class, which is asymptotically stable. The existence also holds for certain power law kinetics where analogues of the DZT are valid [15], with uniqueness in appropriate kinetic analogues of the stoichiometric subspace (see Sect. 6 for a detailed discussion).

We have a further Corollary of Theorem 1:

Corollary 3 (i) A cycle terminal CRN with zero reactant deficiency has high reactant rank, i.e. $\Delta(\mathscr{N})<0$.

(ii) Any $C R N$ with $\delta_{\rho}=\delta=0$ and low or medium reactant $\operatorname{rank}(\Delta(\mathscr{N}) \geq 0)$ has no positive equilibria for any kinetics.

Proof (i) From Theorem 1 (i), we have $0=l+\Delta(\mathscr{N})$ or $l=q-s$. Since $l \geq 1$, we conclude that the network has high reactant rank. Since a weakly reversible network necessarily has high reactant rank, a CRN with $\delta_{\rho}=\delta=0$ and $\Delta(\mathscr{N}) \geq$ 0 is not weakly reversible and it follows from classical results of Feinberg and Horn that it has no positive equilibria for any kinetics.

If a CRN with $\delta_{\rho}=0$ has $\delta>0$, we can combine Theorem 1, with the generalized Feinberg-Horn KSSC (Kinetic and Stoichiometric Subspace Coincidence) Theorem ([2], see also Sect. 5) to derive properties of complex factorizable kinetics on the network in the following proposition:

Proposition 11 (i) If $\mathscr{N}$ has low reactant rank, i.e. $\Delta(\mathscr{N})>0$, then, any positive equilibrium of a (differentiable) complex factorizable kinetics on $\mathscr{N}$ is degenerate. 
(ii) If $\mathscr{N}$ has medium or high reactant rank, i.e. $\Delta(\mathscr{N}) \leq 0$ and point terminal, then, for any (differentiable) factor span surjective kinetics, either $K$ coincides with $S$ ( $t$-minimal case) or non-coincidence may occur (rate constant dependent in the non-t-minimal case), implying degeneracy of positive equilibria.

Proof In (i), we have $\delta=\tau(\mathscr{N})-t_{c}-\Delta(\mathscr{N}) \leq \tau(\mathscr{N})-\Delta(\mathscr{N})<\tau(\mathscr{N})$, since $t_{c} \geq 0$ and $\Delta(\mathscr{N})>0$. The KSSC implies that the kinetic and stoichiometric subspaces do not coincide and hence all positive equilibria are degenerate. In (ii), $\delta=\tau(\mathscr{N})-\Delta(\mathscr{N}) \geq \tau(\mathscr{N})$, since $\Delta(\mathscr{N}) \leq 0$. Hence, the KSSC implies for any factor span surjective kinetics that either $K=S$ ( $t$-minimal case) and $K=S$ is rate constant dependent (non-t-minimal case).

Example 1 The EnvZ-OmpR system, a bacterial signalling system to respond to osmotic pressure:

$$
\begin{aligned}
X & \leftrightarrows X T \rightarrow X_{p} \\
X_{p}+Y & \leftrightarrows X_{p} Y \rightarrow X+Y_{p} \\
X T+Y_{p} & \leftrightarrows X T Y_{p} \rightarrow X T+Y
\end{aligned}
$$

is a point terminal system with $\operatorname{HRR}(q=6>5=s)$. It is $t$-minimal so network deficiency is greater than reactant deficiency. One easily verifies that $\delta=1$, while $\delta_{\rho}=0$.

\subsection{The product subspace and the converse transformation}

We briefly introduce the product subspace of $\mathbb{R}^{\mathscr{S}}$, which is the natural "dual" of the reactant subspace under the converse graph transformation.

Definition 19 The subspace $P$ of $\mathbb{R}^{\mathscr{S}}$ generated by the product complexes (i.e. $<$ $\pi(\mathscr{R})>$ ) is called the product subspace of the CRN. We denote the number of product complexes with $n_{p}$.

Proposition $12 p:=\operatorname{dim} P=\operatorname{dim} \operatorname{Im} N^{+}, \operatorname{dim} \operatorname{ker} N^{+}=r-p$.

Proof Use the converse graph transformation.

Proposition 13 (i) $P+R=\operatorname{Im} Y \operatorname{dim}(P \cap R)=p+q-c($ where $c=\operatorname{dim} \operatorname{Im} Y)$.

(ii) Networks with $P \cap R=0$ form a subset of the class of networks without intermediate complexes, which is characterized by $n_{p}+n_{r}=n$.

Proof (i) An element $x$ in Im $Y$ has the form $x=\sum \tau_{i} y_{i}$ and hence a pre-image $z=\sum \tau_{i} \omega_{y i}$. The claim follows from the fact that each complex is a reactant or product.

(ii) An intermediate complex would be a nonzero element in the intersection. The characterization is given by the formula for the number of intermediate complexes $n_{r}+n_{p}-n$. 


\section{The relationship between the reactant and the stoichiometric subspaces}

The relationship between the reactant subspace $R$ and the stoichiometric subspace $S$ of a CRN in terms of their intersection $R \cap S$ turns out to be important for the kinetic behavior of systems on the network. Hence, we first introduce a classification of CRNs based on the properties of the subspace $R \cap S$. We then introduce the subnetwork of $S$-complexes as an additional tool for analyzing $R \cap S$ and the network classes. The main result of the section (Theorem 2) then characterizes the network classes by equivalences in terms of the containment of $R$ and $S$ in $\operatorname{Im} Y$ and necessary conditions for the subnetwork of $S$-complexes.

\subsection{A classification of CRNs based on the subspace $R \cap S$}

A natural first step for a classification is to differentiate CRNs with trivial intersection, i.e. $R \cap S=0$, from those with non-trivial ones. We denote the former set with TRS, the latter with NRS. We also note that since the (standard) digraph definition excludes loops, i.e. $\operatorname{arcs} y \rightarrow y$, in our considerations, $S \neq 0$. However, $R$ can be trivial, so that such networks belong to TRS.

Two interesting subsets of NRS are defined as follows:

Definition 20 A CRN has a stoichiometry-determined reactant subspace (of type SRS) if its nonzero reactant subspace $R$ is contained in $S$, i.e. $0 \neq R=R \cap S$. It has a reactant-determined stoichiometric subspace (of type RSS) if $S$ is contained in $R$, i.e. $R \cap S=S$. A CRN in SRS $\cap$ RSS has type RES, i.e. $R=S$.

Examples of SRS CRNs are open networks, i.e. $S=R^{\mathscr{S}}$, which include the familiar fully open networks where each species has an outflow reaction. On the other hand, cycle terminal CRNs, including the well-studied weakly reversible networks, belong to RSS. An RSS network with terminal points is given by the following biological system:

Example 2 The MAK system of the following CRN models the calcium dynamics of olfactory cilia [22]:

$$
\begin{aligned}
& A \leftrightarrows B \rightarrow D+B \\
& C+4 D \leftrightarrows E \\
& B+E \rightarrow F \leftrightarrows A+E \\
& D \rightarrow 0
\end{aligned}
$$

The set $\{A, B, C+4 D, E, F, D\}$ is a basis of $R$. On the other hand, since all vectors in the basis for $S=\{B-A, D, E-C-4 D, F-B-E\}$ are linear combinations of the $R$-basis, $S$ is contained in $R$.

Since the kinetic impact of $R$ also depends on its size relative to $S$, we differentiate two further subsets in the following definition: 


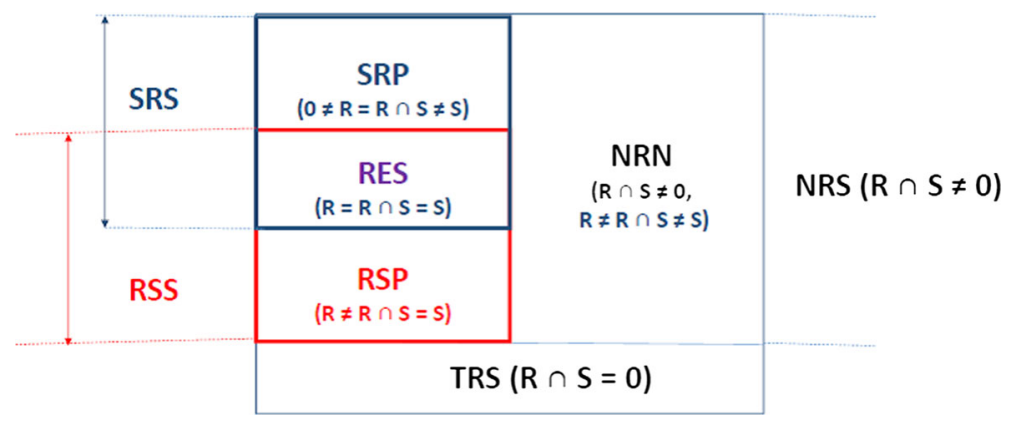

Fig. 1 An overview of the network classes

Definition 21 A CRN is of type SRP if $R$ is a proper subset of $S$, i.e. $0 \neq R=R \cap S \neq$ $S$. Similarly, it is of type RSP if $S$ is a proper subset of $R$, i.e. $R \neq R \cap S=S$.

To complete the classification, we introduce a final network class:

Definition 22 A CRN has a non-trivial and non-containing intersection (of type NRN) if $R \cap S \neq 0$ and $R \neq R \cap S \neq S$. Equivalently, NRN $=$ NRS $\backslash(\mathrm{SRS} \cup \mathrm{RSS})$.

Example 3 The CRN of the EnvZ-OmpR system in Example 1 belongs to this network class. Since $q>s, R \neq R \cap S$. The reaction vector $X T-X$ is in $R \cap S$, but $X_{p}-Y$ is not, hence $0 \neq R \cap S \neq S$.

Figure 1 provides an overview of the network classes.

\subsection{The subnetwork of $S$-complexes}

Our basic definition is:

Definition 23 An $S$-complex of a CRN is a complex which, as a vector in $\mathbb{R}^{\mathscr{S}}$, is contained in the stoichiometric subspace $S$. We denote the subset of $S$-complexes in $\mathscr{C}$ with $\mathscr{C}_{S}$.

Example 4 There are of course CRNs with no $S$-complexes. The network $\mathscr{S}=$ $\left\{X_{1}, X_{2}\right\}, r: X_{1} \rightarrow X_{2}$, has the stoichiometric subspace $<X_{1}-X_{2}>$, and neither $X_{1}$ nor $X_{2}$ is contained in it, hence $\mathscr{C}_{S}=\emptyset$.

On the other hand, we have:

Example 5 If $\mathscr{N}$ is an open network, then $\mathscr{C}_{S}=\mathscr{C}$. This is evident, since $S=\mathbb{R}^{\mathscr{S}}$.

The following Proposition shows that the set of $S$-complexes, when non-empty, has an interesting structure:

Proposition 14 (i) If $y \in S, y^{\prime}$ is linked to $y$, then $y^{\prime} \in S$. In other words, in a linkage class $\mathscr{L}$, either all complexes are in $S$ or none at all. In the first case, we call the linkage class an S-linkage class. 
(ii) If a network $\mathscr{N}$ has a flow, then the linkage class of the zero complex $\mathscr{L}_{0}$ is contained in $\mathscr{C}_{S}$, hence is non-empty.

(iii) If $\mathscr{C}_{S}$ is non-empty, then it is the union of linkage classes, and hence a subnetwork of $\mathscr{N}$, which we denote with $\mathscr{N}_{S}$. The rank (deficiency) of $\mathscr{N}_{S}$ is less (greater) than the sum of the ranks (deficiencies) of the S-linkage classes.

(iv) $\mathscr{N}_{S}$ is an independent subnetwork iff $\mathscr{N}$ has independent linkage classes (ILC), i.e. $\sum \delta_{i}=\delta, i=1, \ldots, l$.

Proof (i) We first show that if $y \in S, y \rightarrow y^{\prime}$, then $y^{\prime} \in S$. Note that $y^{\prime}=$ $y+\left(y^{\prime}-y\right)$, with both summands in $S$, hence also in $S$. Similary, if $y^{\prime} \rightarrow y$, $y^{\prime}=y-\left(y-y^{\prime}\right)$ is also in $S$. Hence any complex in the linkage class of $y$ is also in $S$. We denote the number of $S$-linkage classes with $l_{S}$. We have $0 \leq l_{S} \leq l$.

(ii) Since 0 is in $S$, all of the complexes in its linkage class are also in $S$.

(iii) The first statement is clear, and since the complexes of a linkage class also uniquely determine the reactions among them, to determine the subnetwork, we set $R^{\prime}$ as the union of all reactions between $S$-complexes. Recall that, as formalized by Joshi-Shiu, we take as complexes those occurring in the reactions in $R^{\prime}$-these are precisely the $S$-complexes, and as species those occurring in the $S$-complexes.

(iv) The proof follows the fact that both $\mathscr{N}_{S}$ and its complement are unions of linkage classes.

An immediate consequence is the following corollary:

Corollary 4 (The Single Linkage Class $\mathscr{N}_{S}$ Alternative). If a network has a single linkage class, then either $\mathscr{N}=\mathscr{N}_{S}$ or $\mathscr{N}_{S}=\emptyset$. If the network contains the zero complex, then $\mathscr{N}=\mathscr{N}_{S}$.

We note some properties of the reactant and stoichiometric subspaces of the subnetwork of $S$-complexes:

Proposition 15 Let $R_{S}$ and $S_{S}$ be the reactant and stoichiometric subspaces of $\mathscr{N}_{S}$, respectively. Then:

(i) $R_{S} \subset S_{S}$, and

(ii) For $q_{S}:=\operatorname{dim} R_{S}$ and $s_{S}:=\operatorname{dim} s_{S}$, we have $q_{S} \leq \sum q_{S, i}$ and $s_{S} \leq \sum s_{S, i}$, respectively, where $i$ runs over all linkage classes in $\mathscr{N}_{S}$. Equality holds if the network has the ILC property.

Proof (i) By definition, all complexes in $\mathscr{N}_{S}$ will be in $S_{S}$ so that $\left(\mathscr{N}_{S}\right)_{S}=\mathscr{N}_{S}$, implying $R_{S} \subset S_{S}$. (ii) This follows directly from the usual rank inequalities for linkage classes and networks for $q$ and $s$.

\subsection{Characterization of the $R \cap S$-based network classes}

The following characterization in terms of containment of $R$ and $S$ in $\operatorname{Im} Y$ and the subnetwork of $S$-complexes is the basis for the connections to kinetic properties discussed in Sects. 5 and 6. 
Theorem 2 Let $Y$ be the map of complexes of a network $\mathscr{N}$ with subnetwork $\mathscr{N}_{S}$ of S-complexes.

(i) $\mathscr{N}$ is $S R S \Leftrightarrow \operatorname{Im} Y=S \Leftrightarrow c=s$. Furthermore, $\mathscr{N}$ is $S R S \Rightarrow \mathscr{N}=\mathscr{N}_{S}$.

(ii) $\mathscr{N}$ is $R S S \Leftrightarrow \operatorname{Im} Y=R \Leftrightarrow c=q$. Furthermore, $\mathscr{N}$ is $R S S \Rightarrow$ either $\mathscr{N}=$ $\mathscr{N}_{S}(R E S)$ or $\mathscr{N} \neq \mathscr{N}_{S}(R S P)$.

(iii) $\mathscr{N}$ is TRS $\Leftrightarrow \operatorname{Im} Y$ is a direct sum of $R$ and $S \Leftrightarrow c=q+s$. Furthermore, $\mathscr{N}$ is $T R S \Rightarrow \mathscr{N} \neq \mathscr{N}_{S}$ and, if $\mathscr{N}$ has no inflow reaction, $\mathscr{N}_{S}=\emptyset$.

(iv) $\mathscr{N}$ is $N R N \Rightarrow c<q+s<2 c$. Furthermore, $\mathscr{N}$ is $N R N \Rightarrow \mathscr{N} \neq \mathscr{N}_{S}$.

Proof For both (i) and (ii), the converse $(\Leftarrow)$ is immediate since $S$ and $R$ are contained in $\operatorname{Im} Y$. To show $(\Rightarrow)$ for (i), write $y^{\prime}=\left(y^{\prime}-y\right)+y$, and from which $P \subset R$ will follow. Since $P+R=\operatorname{Im} Y$ according to Proposition 13, we obtain $R=\operatorname{Im} Y$. The argument is analogous for $(\Rightarrow)$ of (ii). If $c=\operatorname{dim} \operatorname{Im} Y=\operatorname{rank} Y$, then (i) is also equivalent to $q=c$ and (ii) to $s=c$.

We now observe that the necessary conditions $\mathscr{N} \neq \mathscr{N}_{S}$ in (ii), (iii) and (iv) altogether are equivalent to $\mathscr{N}=\mathscr{N}_{S} \Rightarrow \mathscr{N}$ is SRS. Hence, we next prove that $\mathscr{N}$ is $\mathrm{SRS} \Leftrightarrow \mathscr{N}=\mathscr{N}_{S}$.

For $(\Rightarrow)$, all complexes of the network are in $S$, including $\rho(R)$, the generators of $R$, hence $R \subset S$. For $(\Leftarrow)$, by assumption, each reactant complex is in $S$. Now, since in each linkage class there is at least one reactant complex, then each linkage class is contained in $\mathscr{N}_{S}$ and hence the whole network.

To prove (iii), we first show that $\operatorname{Im} Y=R+S$. Any complex is either a reactant complex or a product only complex. The former is clearly in the sum, in the latter case write $y^{\prime}=y+\left(y^{\prime}-y\right)$. Hence $\operatorname{Im} Y$ is the direct sum of $R$ and $S$, and the dimension equation follows.

Suppose the complex $z \in \mathscr{N}_{S}$. If $z$ is a reactant complex, then we are done. If it is a product, there is a reaction $y \rightarrow z$, so that the nonzero reactant complex is in $S$ too, being in the same linkage class. This shows that $R \cap S \neq 0$.

Finally, to show (iv), since $R \cap S \neq 0, \operatorname{dim} R+\operatorname{dim} S>\operatorname{dim}(R+S)=\operatorname{dim} \operatorname{Im} Y$, hence $q+s>c$. On the other hand, since the intersection is not equal to $R$ or $S, s<c$ and $q<c$, so that $q+s<2 c$. This completes the proof of the Theorem.

Remark 5 The simple example $0 \rightarrow X$ shows that the hypothesis $\mathscr{N}$ has no inflow reaction is essential in Theorem 2 (iii).

Example 6 The EnvZ-OmpR model from Example 1 is a counterexample to the converse of Theorem 2 (iii). One easily checks that it has no inflow reaction, $\mathscr{N}_{S}=\varnothing$, but $S \cap R$ has at least $\operatorname{dim}=1$ since $X T-X$ is contained in it.

\section{The coincidence of the reactant and the kinetic subspace of a chemical kinetic system}

The containment of a system's kinetic subspace $K$ in the stoichiometric subspace $S$ of its underlying network expresses an important connection between system behavior and network structure. $K \neq S$ or their non-coincidence, for example, implies that all of the system's equilibria are degenerate. M. Feinberg and F. Horn were the first to 
study the coincidence of the kinetic and the stoichiometric subspaces in 1977[7]. Their main result, which we call the Feinberg-Horn KSSC (Kinetic and Stoichiometric Subspace Coincidence) Theorem identified network properties sufficient for coincidence in MAK systems:

Theorem 3 If $K$ is the kinetic subspace of a MAK system, and:

(i) if $t-l=0$, then $K=S$.

(ii) if $t-l>\delta$, then $K \neq S$.

(iii) if $0<t-l \leq \delta, K=S$ or $K \neq S$ is rate constant dependent.

Forty years later, Arceo et al [2] extended the KSSC Theorem to the set of complex factorizable kinetics (CFK) using the concept of span surjectivity as follows:

Theorem 4 For a complex factorizable system on a network $\mathscr{N}$,

(i) if $t-l>\delta$, then $K \neq S$.

(i') if $0<t-l \geq \delta$, and a positive steady state exists, then $K \neq S$. In fact $\operatorname{dim} S-\operatorname{dim} K \geq t-l-\delta+1$.

if the system is also factor span surjective and

(ii) if $t-l=0$ (i.e. $\mathscr{N}$ is $t$-minimal), then $K=S$.

(iii) if $0<t-l \leq \delta$, then it is rate constant dependent whether $K=S$ or not.

\subsection{RKS kinetics}

To better understand kinetics with the KRSC property, i.e. $K=R$, we study their superset of kinetics with $K \subset R$. This containment in contrast to that in $S$ is true only for certain kinetics, which we denote as RKS kinetics:

Definition 24 A chemical kinetics on a $\mathrm{CRN}$ has a reactant-determined kinetic subspace (of type RKS) if $K$ is contained in $R$.

Determining the containment of $K$ in $R$ is in general not easy since one does not have a generic set of generators for $K$. A useful approach is to determine a superset of $K$, e.g. $S$, and study the relationship of the superset to $R$. The following proposition, which demonstrates the strong dependence of RKS kinetics on network type, is derived using this approach.

Proposition 16 (i) Any kinetics on an RSS network has the RKS property.

(ii) On a TRS network (i.e. $R \cap S=\emptyset$ ), there are no $R K S$ kinetics.

Proof (i) $K \subset S$ and $S \subset R$ (RSS property) $\Rightarrow K \subset R$. (ii) Note that for any kinetics, $\operatorname{dim} K \geq 1$. For an RKS kinetics, $K=K \cap S \subset R \cap S=0$ (NRS property), implying that no such kinetics exists.

Another useful superset of $K$ is $K^{+}+K^{-}$, where $K^{i}=\operatorname{span}\left(\operatorname{Im} f^{i}\right)$ and $f^{i}=$ $Y I_{a}^{i} K$ for $i \in\{+,-\}$. In fact, we have the following characterization of an RKS kinetics: 
Proposition $17 K$ is an RKS kinetics $\Leftrightarrow K^{+}+K^{-} \subset R$.

Proof Recall that $f(x)=f^{+}(x)-f^{-}(x)$ for all $x \in \Omega_{K}$, so $K \subset K^{+}+K^{-}$for any kinetics, and $(\Leftarrow)$ immediately follows. On the other hand, if $K \subset R$, each $f(x)$ is also in $R$ implying $f^{+}(x)=f(x)+f^{-}(x)$ is also in $R$. Since the $f^{+}(x)$ generate $K^{+},(\Rightarrow)$ follows.

Remark 6 Note that the subspace $K^{+} \cap K^{-}$is of interest in determining positive equilibria because it contains all the common values an equilibrium of $f$ can attain under $f^{+}$and $f^{-}$, since $f(x)=0 \Leftrightarrow f^{+}(x)=f^{-}(x)$. In particular, for any kinetics, $K^{+} \cap K^{-}=0$ implies $E_{+}(\mathscr{N}, K)=E_{+}\left(\mathscr{N}, f^{+}\right) \cap E_{+}\left(\mathscr{N}, f^{-}\right)$, where $E_{+}$is the set of positive equilibria of $\mathscr{N}$. Also, if $K$ is an RKS kinetics, then all these values are in $R$.

In general, little is known about the dimension of the kinetic subspace $K$, only the fact that $1 \leq \operatorname{dim} K \leq s$. For RKS kinetics, we can improve the upper bound:

Proposition 18 For an RKS kinetics, $\operatorname{dim} K \leq q+s-c \leq \min (q, s)$.

Proof A kinetics is RKS $\Leftrightarrow K \subset R \cap S$. Since $\operatorname{Im} Y=R+S$, it follows from linear algebra, that $\operatorname{dim}(R \cap S)=q+s-c$. Since $q-c \leq 0$ and $s-c \leq 0$, it follows that $s+q-c \leq s$ and $q+s-c \leq q$, respectively.

\subsection{RKS kinetics and factor span surjectivity}

For a complex factorizable kinetics, if $K \neq S$ and $S$ is not a subset of $R$, there is still a chance that $K$ is contained in $R$. A sufficient condition would be the existence of a positive vector $k$ such that $\operatorname{Im} Y A_{k}$ is contained in $R$.

The following two propositions illustrate this and also elucidate further connections between RKS kinetics and span surjectivity.

Proposition 19 Let $\mathscr{N}$ be a terminal point containing network. If for a CF kinetics with factorization equals $I_{k} \Psi_{k}$ and with Im $Y A_{k}$ contained in $R$, then the kinetics has the RKS property.

To apply this proposition, one can start from a basis of $\mathbb{R}^{\mathscr{C}}$ (e.g. a basis of ker $A_{k}$ and $\left(\operatorname{ker} A_{k}\right)^{\perp}$ ), then apply consecutively $A_{k}$ and $Y$ to obtain a generating set for $\operatorname{Im} Y A_{k}$. Then one can apply an appropriate software routine to check if it is contained in $R$.

For a factor span surjective kinetics, one obtains a converse:

Proposition 20 Let $\mathscr{N}$ be a terminal point containing network. If for a factor span surjective kinetics Im $Y A_{k}$ is not contained in $R$, then it does not have the RKS property.

Proof We showed in [2] that for factor span surjective kinetics, $K=\operatorname{Im} Y A_{k}$.

\subsection{The kinetic and reactant subspace coincidence (KRSC) theorem}

Our main result provides further striking connections between the RKS property and factor span surjectivity: 
Theorem 5 (Kinetic and Reactant Subspace Coincidence Theorem). Let $c=$ $\operatorname{dim} \operatorname{Im} Y$ and $s, q$ the rank and reactant rank of the network $\mathscr{N}$, respectively. For a complex factorizable system on $\mathscr{N}$,

(i) If $c-s>0$, then $K \neq R$.

(ii) If $c-s=0$ and $c-q>0, t-l=0$ and the system is factor span surjective, then $K \neq R$.

(iii) If $c-s=0$ and $c-q>0, t-l>0$ and the system is factor span surjective, then whether $K=R$ or not, is rate constant and equilibrium existence dependent.

(iv) If $c-s=0$ and $c-q=0, t-l=0$ and the system is factor span surjective, then $K=R$.

Proof (i) $c-s>0 \Leftrightarrow S$ is a proper subset of $\operatorname{Im} Y$. It follows from Theorem 2 that $\mathscr{N}$ is not an SRS network, i.e. $R$ is not contained in $S \Leftrightarrow S \cap R \neq R$. If $K=R$, then $K=K \cap S=R \cap S \neq R$, a contradiction. Hence $K \neq R$.

(ii) $c-s=0$ and $c-q>0$ imply that if $K=S$, then $K \neq R$. Since the system is factor span surjective, statement (2) of the KSSC implies this for $t-l=0$.

(iii) For $c-s=0$ and $c-q>0$ and $t-l>0$, statement (3) of the KSSC implies that $K=S$ is rate constant and equilibrium existence dependent, and hence this also holds for $K$ and $R$ coincidence.

(iv) $c-s=0$ and $c-q=0 \Leftrightarrow S=R$. Statement (2) of the KSSC again implies that $K=S$, hence $K=R$.

Remark 7 (i) Theorem 5 (i) depends only on network properties just as in KSSC. In contrast to KSSC (where the subspace $\operatorname{Im} Y A_{k}$ plays a key role in the derivation), it is based only on the intersection $R \cap S$ and hence is also valid for non-complex factorizable kinetics.

(ii) Theorem 5 (iv) uses the Feinberg-Horn Theorem to achieve $K=S$. Hence for $R=S$, the assertion is also valid for other kinetics with KSSC such as span surjective kinetics. These are non-complex factorizable on networks with no inflow-branching and non-inflow branching (which is the branching type " $\mathrm{S}$ " in [2]).

In summary, we conclude that the coincidence of $K$ and $R$ can occur only on SRS networks, but it has a dual character: coincidence on an SRP network implies that $K \neq S$, and hence all the equilibria of a complex factorizable kinetics with differentiable factor map are degenerate. On the other hand, coincidence on an RES network implies $K=S$ and hence the possibility of the existence of non-degenerate equilibria.

\section{The kinetic flux subspace of power law systems}

In the Deficiency Zero and Deficiency One Theorems for MAK systems, the stoichiometric subspace $S$ plays an important kinetic role in two ways: its orthogonal complement is used to parametrize the set of positive equilibria and each stoichiometric class intersects the equilibria set in a unique point. More precisely, one has: 
(a) $x * \in E_{+}$implies that $E_{+}=\left\{x \in \mathbb{R}_{>}^{\mathscr{S}} \mid \log (x)-\log (x *) \in S^{\perp}\right\}$, and

(b) $\left|E_{+} \cap \mathscr{P}\right|=1$ for each positive stoichiometric class $\mathscr{P}$.

Hence, in order to establish analogues for the Low Deficiency Theorems for power law kinetic systems, a "kinetic analogue" of the stoichiometric subspace needs to be identified. In their work on Generalized Mass Action Kinetic (GMAK) systems, a superset of PL-RDK systems, Müller and Regensburger [12] introduced the kinetic order subspace $\widetilde{S}_{\mathrm{MR}}$ as such an analogue for cycle terminal networks and derived a Deficiency Zero Theorem for systems with zero kinetic deficiency, which form a subset of zero deficiency systems.

The Müller-Regensburger kinetic order subspace is sufficient for zero deficiency systems since for such systems, the positive equilibria set is non-empty if and only if the network is weakly reversible, and such networks are cycle terminal. However, for the Deficiency One Theorem, non-cycle terminal networks, i.e. networks with terminal points, also need to be considered. The main result of this section is the construction of the kinetic flux subspace $\widetilde{S}$ for any PL-RDK system with the following properties:

(a) $\widetilde{S}=S$ for the subset of MAK systems.

(b) $\widetilde{S}=\widetilde{S}_{\mathrm{MR}}$ for a PL-RDK system on a cycle terminal network.

We illustrate the kinetic role of $\widetilde{S}$ with examples of PL-RLK systems, a set of PL-RDK systems with "linearly independent" kinetics in Sect. 7. In [17], a subspace of $\widetilde{S}$, which in many cases coincides with it, is used for the parametrization and uniqueness results in the Low Deficiency Theorems derived there for a superset of PL-RLK systems.

\subsection{The kinetic reactant subspace and T matrix of a PL-RDK system}

In their more geometric approach to power law kinetics in [12], Müller and Regensburger introduced the concept of a generalized chemical reaction network (GCRN) by specifying a map $\tilde{y}: \rho(\mathscr{R}) \rightarrow \mathbb{R}^{\mathscr{S}}$, assigning to a reactant complex $y$ the row of kinetic order matrix of its reaction, which they called its kinetic complex $\tilde{y}(y)$. The kinetic complexes appear in a further construct in their paper, namely as columns of the $m \times n$ matrix $\widetilde{Y}$ corresponding to reactant complexes together with zero columns for non-reactant complexes (i.e. terminal points). In this section, we refine their concepts by (1) considering the subspace generated by the kinetic complexes and (2) truncating away the zero columns of $\widetilde{Y}$. These two changes enable a stronger "reactant-oriented" conceptual focus.

\subsubsection{The kinetic reactant subspace of a power law system}

In analogy to the reactant subspace of the network, we now define:

Definition 25 The kinetic reactant subspace $\widetilde{R}$ is the subspace generated by the kinetic complexes in $\mathbb{R}^{\mathscr{S}}$. The $\operatorname{dim} \widetilde{R}$ is called the kinetic reactant rank and elements of $\mathbb{R}^{\mathscr{S}} / \widetilde{R}$ the kinetic reactant classes.

For a MAK system, $\tilde{y}=i d$ and hence $\widetilde{R}=R$. For a cycle terminal network, Müller and Regensburger introduced the kinetic analogue of the stoichiometric subspace of the network: 
Definition 26 For a cycle terminal network $\mathscr{N}$, the kinetic order subspace $\widetilde{S}_{\mathrm{MR}}$ is the span of the fluxes of the kinetic complexes, i.e.

$$
\widetilde{S}_{\mathrm{MR}}:=\operatorname{span}\left\{\tilde{y}\left(y^{\prime}\right)-\tilde{y}(y) \mid y \rightarrow y^{\prime} \in \mathscr{R}\right\} .
$$

We observe that for any PL-RDK system, $\widetilde{S}_{\text {MR }} \subset \widetilde{R}$. This suggests that a generalization to non-cycle terminal networks should also be contained in $\widetilde{R}$. For a MAK system on a cycle terminal network, $\widetilde{S}_{\mathrm{MR}}=S \subset R$. With these considerations in mind, we reformulate our task - on an RSS network (i.e. $S \subset R$ ), identify a subspace of $\widetilde{R}$ with the following properties:

(i) $\underset{S}{\widetilde{S}}=\underset{S}{S}$ for the subset of MAK systems,

(ii) $\widetilde{S}=\widetilde{S}_{\mathrm{MR}}$ for a PL-RDK system on a cycle terminal network.

\subsubsection{The T matrix of a PL-RDK system}

We recall the definition of the $m \times n$ matrix $\widetilde{Y}$ from [13]: for a reactant complex, the column of $\widetilde{Y}$ is the transpose of the kinetic order matrix row of the complex's reaction, otherwise (i.e. for a terminal point), the column is 0 .

Definition 27 The $T$ matrix of a PL-RDK system is formed by truncating away the columns of the terminal points in $\widetilde{Y}$, obtaining an $m \times n_{r}$ matrix. The corresponding linear map $T: \mathbb{R}^{\rho(\mathscr{R})} \rightarrow \mathbb{R}^{\mathscr{S}}$ maps $\omega_{\rho(r)}$ to $\left(F_{r}\right)^{T}$. The $T$ matrix is the kinetic analog on of the map $Y_{\text {res }}: \mathbb{R}^{\rho(\mathscr{R})} \rightarrow \mathbb{R}^{\mathscr{S}}$ on network (or MAK system) level. Just as $\operatorname{Im} Y_{\text {res }}=R, \operatorname{Im} T=\widetilde{R}$.

\subsection{Kernel-aligned PL-RDK systems on RSS networks}

Let $\mathscr{N}$ be an RSS network, $K$ a PL-RDK kinetics on $\mathscr{N}$. If the map of sets $\tilde{y}$ can be extended to a linear map of subspaces $R \rightarrow \widetilde{R}$, then the image $\widetilde{y}(S)$ would be a candidate for the subspace we are looking for. A subset of PL-RDK enabling this is easily identified:

Definition 28 A PL-RDK kinetics is $Y$-kernel aligned (of type YKA) if $\operatorname{ker} Y_{\text {res }} \subset$ $\operatorname{ker} T$.

Proposition 21 For a PL-YKA system, the linear map $\tilde{y}: R \rightarrow \widetilde{R}$ is well defined (and surjective). If $\mathscr{N}$ has zero reactant deficiency, then $\mathscr{P} \mathscr{L}-\mathscr{R} \mathscr{D} \mathscr{K}(\mathscr{N})=$ $\mathscr{P} \mathscr{L}-\mathscr{Y} \mathscr{K} \mathscr{A}(\mathscr{N})$.

Proof We extend $\tilde{y}$ linearly to $R \rightarrow \widetilde{R}$, i.e. for $y=\sum \alpha_{i} y_{i}$ with $y_{i} \in \rho(R), \tilde{y}(y):=$ $\sum \alpha_{i} \tilde{y}\left(y_{i}\right)$. If $y=\sum \beta_{i} y_{i}$, then $\sum\left(\alpha_{i}-\beta_{i}\right) \omega_{y_{i}} \in \operatorname{ker} Y_{\text {res }}$. Since the kinetics is YKA, $T\left(\sum\left(\alpha_{i}-\beta_{i}\right) \omega_{y_{i}}\right)=\sum\left(\alpha_{i}-\beta_{i}\right) T\left(\omega_{y_{i}}\right)=\sum\left(\alpha_{i}-\beta_{i}\right) \widetilde{y}(y i)=0$ and the map is welldefined and surjective. Furthermore, $\mathscr{N}$ has zero reactant deficiency $\Leftrightarrow \operatorname{ker} Y_{\text {res }}=0$, which implies $\mathscr{P} \mathscr{L}-\mathscr{R} \mathscr{D} \mathscr{K}(\mathscr{N})=\mathscr{P} \mathscr{L}-\mathscr{Y} \mathscr{K} \mathscr{A}(\mathscr{N})$.

Definition 29 For a PL-YKA system on an RSS network, the kernel-aligned kinetic flux subspace $\widetilde{S}_{K}:=\tilde{y}(S)$. 
Clearly, any MAK system on an RSS network is YKA, so that $\widetilde{S}_{K}=S$. For a cycle terminal network, since $\tilde{y}\left(y_{j} y_{i}\right)=\tilde{y}\left(y_{j}\right) \tilde{y}\left(y_{i}\right)$ for reactant complexes $y_{i}, y_{j}$, we have $\widetilde{S}_{K}=\widetilde{S}_{\text {MR }}$. Hence, the kernel-aligned kinetic flux subspace has both desired properties for a subspace of $\widetilde{R}$. However, as the following example shows, there are RSS networks where $\mathscr{P} \mathscr{L}-\mathscr{R} \mathscr{D} \mathscr{K}(\mathscr{N}) \neq \mathscr{P} \mathscr{L}-\mathscr{Y} \mathscr{K} \mathscr{A}(\mathscr{N})$, so that $\widetilde{S}_{K}$ does not provide a complete solution for our search.

Example 7 The RSS network given by $X 1+X 2 \rightarrow X 1$ and $2 X 1+2 X 2 \rightarrow 2 X 1$ has a 1 -dim $\operatorname{ker} Y_{\text {res }}$. On the other hand, the PL-RDK system with $T$ matrix

$$
T=\left[\begin{array}{cc}
0.1 & 0 \\
0 & 0.2
\end{array}\right]
$$

has $\operatorname{ker} T=0$, so it is not YKA on the network above.

\subsection{The kinetic flux subspace of a PL-RDK system}

The observation that for an RSS network with zero reactant deficiency, $\tilde{y}(S)=T$ 。 $Y_{\text {res }}^{-1}(S)$, where $Y_{\text {res }}^{-1}$ is the inverse map, suggested the correct general solution for the problem:

Definition 30 For a PL-RDK kinetics on an RSS network, the kinetic flux subspace $\widetilde{S}$ is the subspace $T\left(Y_{\text {res }}^{-1}(S)\right)$ of $\widetilde{R}$. We denote by $\widetilde{S}=\operatorname{dim} \widetilde{S}$ the kinetic rank.

In the definition, $Y_{\text {res }}^{-1}(S)$ is the pre-image of $S$ in $R^{\rho(\mathscr{R})}$, which is also a linear subspace. We note that an RES network implies $\widetilde{S}=\widetilde{R}$ and $\widetilde{s}=\widetilde{q}$. This follows immediately from $Y_{\text {res }}^{-1}(R)=R^{\rho(\mathscr{R})}$ and $T\left(R^{\rho(\mathscr{R})}\right)=\widetilde{R}$.

Remark 8 Applying the Nullity Theorem to the restriction of $T$ to $Y_{\text {res }}^{-1}(S)$ shows that $\widetilde{S}$ is isomorphic to $Y_{\text {res }}^{-1}(S) / Y_{\text {res }}^{-1}(S) \cap \operatorname{ker} T$. This means that the ambiguities with ker $T$ are factored away.

We now prove our main result in Sect. 6:

Theorem 6 Let $(\mathscr{N}, K)$ be a PL-RDK system on an RSS network $\mathscr{N}$ with kinetic flux subspace $\widetilde{S}$.

(i) If $\mathscr{N}$ is cycle terminal and $\widetilde{S}_{M R}$ is its kinetic order subspace, then $\widetilde{S}=\widetilde{S}_{M R}$.

(ii) For any $M A K$ system on $\mathscr{N}, \widetilde{S}=S$.

(iii) For a PL-YKA kinetics, $\widetilde{S}_{K}=\widetilde{S}$.

Proof (i) The equality $\tilde{y}(\rho(r))=T\left(\omega_{\rho(r)}\right)$ holds for any PL-RDK system. For a cycle terminal network, the coincidence of $\widetilde{S}=\widetilde{S}_{\mathrm{MR}}$ rests on the fact that $z=\tilde{y}\left(\rho\left(r^{\prime}\right)\right)-\tilde{y}(\rho(r))$ is a generator of the kinetic order subspace iff $z=T\left(\omega_{\rho\left(r^{\prime}\right)}-\omega_{\rho(r)}\right)$ is a generator of $T\left(Y_{\text {res }}^{-1}(S)\right)$.

Let $x \in \widetilde{S}_{\mathrm{MR}}$ and write $x=\sum \alpha_{i}\left(\tilde{y}\left(y_{i}^{\prime}\right)-\tilde{y}\left(y_{i}\right)\right)$, where the sum is over all reactions, which are indexed by $i=1, \ldots, r$. Substituting as above, $x=$ 
$\sum \alpha_{i}\left(T\left(\omega_{y_{i}^{\prime}}\right)-T\left(\omega_{y_{i}}\right)\right)=T\left(\sum \alpha_{i}\left(\omega_{y_{i}^{\prime}}-\omega_{y_{i}}\right)\right)$ since the network is cycle terminal. Since $Y\left(\sum \alpha_{i}\left(\omega_{y_{i}^{\prime}}-\omega_{y_{i}}\right)\right)=\sum \alpha_{i}\left(y_{i}^{\prime}-y_{i}\right)$ then $z=\sum \alpha_{i}\left(\omega_{y_{i}^{\prime}}-\omega_{y_{i}}\right) \in$ $Y_{\text {res }}^{-1}(S)$ and $T(z)=x$.

Let $x \in \widetilde{S}$. Then $x=T(z), z \in Y_{\text {res }}^{-1}(S)$. Write $z=\sum \alpha_{i}\left(\omega_{y_{i}^{\prime}}-\omega_{y_{i}}\right)$. Then $x=$ $T(z)=T\left(\sum \alpha_{i}\left(\omega_{y_{i}^{\prime}}-\omega_{y_{i}}\right)\right)=\sum \alpha_{i}\left(T\left(\omega_{y_{i}^{\prime}}\right)-T\left(\omega_{y_{i}}\right)\right)=\sum \alpha_{i}\left(\tilde{y}\left(y_{i}^{\prime}\right)-\tilde{y}\left(y_{i}\right)\right)$, since the network is cycle terminal. The last term is clearly in $\widetilde{S}_{\mathrm{MR}}$.

(ii) This is straightforward from the surjectivity of $Y_{\text {res }}$.

(iii) An element $x$ of $\widetilde{S}_{K}$ has form $\widetilde{y}\left(\sum \alpha_{i}\left(y_{i}^{\prime}-y_{i}\right)\right)=\sum \alpha_{i}\left(\widetilde{y}\left(y_{i}^{\prime}\right)-\widetilde{y}\left(y_{i}\right)\right)$, since $\tilde{y}$ is linear and hence in the kinetic flux subspace $\widetilde{S}$. The same argument as in (i) establishes that each $x$ in the kinetic flux space is also in $\tilde{y}(S)$.

We now present a comparison with another subspace of $\widetilde{R}$ to broaden our understanding of $\widetilde{S}$.

In [16], D. Talabis introduced for any PL-RDK system the map $\widetilde{F}_{T}:=T \circ \operatorname{proj}_{R^{\prime}} \circ I_{a}$ where $R^{\prime}=\mathbb{R}^{\rho(\mathscr{R})}$, which we now call the "truncated kinetic map".

Definition 31 The image of $\widetilde{F}_{T}$ is called the truncated kinetic order subspace $\widetilde{S}_{T}$ of the PL-RDK system. For a MAK system, it is a subspace of $R$ and denoted with $S_{T}$ and called the truncated stoichiometric subspace.

The truncated kinetic order subspace clearly coincides with the Müller-Regensburger kinetic order space for cycle terminal networks, the projection map being the identity. On the other hand, the following example provides a network with $S \neq S_{T}$.

Example 8 A simple example from [4] is the following:

$$
\begin{aligned}
& R 1: 2 X 1 \rightarrow X 1+X 2 \\
& R 2: X 1+X 2 \rightarrow 2 X 1 \\
& R 3: X 1+X 2 \rightarrow 2 X 2
\end{aligned}
$$

It is $t$-minimal, has $\delta=1$ and $\delta_{\rho}=0$. For any MAK system, $\widetilde{S}=S=\langle X 1-X 2\rangle$ is one-dimensional while $\widetilde{R}=R=\langle X 1+X 2,2 X 1\rangle=\mathbb{R}^{2}$.

So the condition $S=S_{T}$ must be explicitly required if the truncated kinetic order subspace should qualify as an extension of the Müller-Regensburger construct. $S=S_{T}$ immediately implies that the network is RSS, so we can compare the truncated kinetic order subspace $\widetilde{S}_{T}$ with the kinetic flux subspace $\widetilde{S}$.

Proposition 22 Let $\mathscr{N}$ be an RSS network with stoichiometric subspace $S$ and kinetic flux subspace $\widetilde{S}$. If $S=S_{T}$, for a PL-RDK kinetics, $\widetilde{S}=\widetilde{S}_{T}+T\left(\operatorname{ker} Y_{\text {res }}\right) . \widetilde{S}=\widetilde{S}_{T}$ iff the kinetics is PL-YKA.

Proof $S=S_{T}$ implies $Y_{\text {res }}^{-1}(S)=Y_{\text {res }}^{-1}\left(S_{T}\right)$. An element of $I_{a, \rho(r)}\left(\mathbb{R}^{\mathscr{R}}\right)$ has the form $z=\sum \alpha_{i}\left(\omega_{y_{i}^{\prime}}-\omega_{y_{i}}\right)+\sum \alpha_{i}\left(-\omega_{y_{i}}\right)$ with the first sum over all non-terminal and the second over all terminal reactions of the network, is in $Y_{\text {res }}^{-1}\left(S_{T}\right)$. This is because 
$s:=Y_{\text {res }}(z)=\sum \alpha_{i}\left(y_{i}^{\prime}-y_{i}\right)+\sum \alpha_{i}\left(-y_{i}\right)$, is an element of $S_{T}$. If $Y_{\text {res }}\left(z^{\prime}\right)=s$ for another $z^{\prime} \in Y_{\text {res }}^{-1}\left(S_{T}\right)$, then $Y_{\text {res }}\left(z-z^{\prime}\right)=0$. In other words, $Y_{\text {res }}^{-1}\left(S_{T}\right)=I_{a, \rho(r)}\left(\mathbb{R}^{\mathscr{R}}\right)+$ $\operatorname{ker} Y_{\text {res. }}$. If we apply $T$ to both sides, we obtain the claim. The second statement follows immediately from the definition of PL-YKA.

Remark 9 (i) If $\mathscr{N}$ is cycle terminal, $S_{T}=S$ and $\widetilde{S}_{T}=\widetilde{S}_{\mathrm{MR}}$ (since $I_{a, \rho(r)}=I_{a}$ ) and hence $\widetilde{S}_{T}=\widetilde{S}$. It follows from Proposition 11 that, in this case, $T\left(\operatorname{ker} Y_{\text {res }}\right) \subset \widetilde{S} T$.

(ii) The YKA-property is sufficient for $\widetilde{S}_{T}=\widetilde{S}$, but not necessary. On a cycle terminal network with $n_{r} \leq m$ and no zero complex, there are PL-RDK kinetics with $\operatorname{ker} T=0$, so if its reactant deficiency is greater than 0 , then those kinetics are not YKA. Nevertheless, as noted in (i) $\widetilde{S}_{T}=\widetilde{S}$ for any PL-RDK system.

\section{Conclusion}

In conclusion, we summarize our main results and outline some perspectives for further research.

1. We derived a formula for the difference between (network) deficiency and reactant deficiency (Theorem 1), which we use to determine existence and characteristics of positive equilibria of kinetics on CRNs with zero reactant deficiency.

2. We characterized the network classes (Theorem 2) in terms of the containment of $R$ and $S$ in $\operatorname{Im~} Y$ and the subnetwork of $S$-complexes, a new tool that we introduce. The network classes play an important role in the connections between $\mathrm{R}$ and the system kinetics.

3. We provided an analogue of the Feinberg-Horn Theorem on the coincidence of the kinetic and stoichiometric subspaces for the kinetic and reactant subspaces of complex factorizable kinetics (Theorem 5). The striking fact that $K$ and $R$ coincidence can occur only in the network class with $R \cap S=R$ exemplifies the role of the network classes in the connections between $R$ and kinetics.

4. We constructed the "kinetic flux subspace" and showed in Theorem 6 that it satisfied the requirements of coincidence with the stoichiometric subspace for MAK systems and with the kinetic order subspace of Müller-Regensburger for PL-RDK systems on cycle terminal networks. A key observation is that these requirements imply that the construction can occur only when $R \cap S=S$, again emphasizing the role of the network classes.

Further research should explore applying the results to existing models to uncover further connections between R and system kinetics. In a companion paper [1], we have carried this out for biochemical systems modeled in the $S$-system formalism. Comparative analysis of models of the earth's carbon cycle has provided further interesting results regarding CRNs with zero reactant deficiency [9].

Acknowledgements Open access funding provided by Max Planck Society. The authors thanks Dylan Talabis for computational support in several examples in the paper. ARL held a research fellowship from De La Salle University and would like to acknowledge the support of De La Salle University's Research Coordination Office. ECJ is supported by the UP-OVPAA ECWRG (2016-1-010) and IMSP, UPLB Collaborative Research Grant. ERM acknowledges the support of De La Salle University's OVCAA for a Visiting Professorship in November-December 2016 during which some of this research was done. 
Open Access This article is distributed under the terms of the Creative Commons Attribution 4.0 International License (http://creativecommons.org/licenses/by/4.0/), which permits unrestricted use, distribution, and reproduction in any medium, provided you give appropriate credit to the original author(s) and the source, provide a link to the Creative Commons license, and indicate if changes were made.

\section{References}

1. C. Arceo, E. Jose, A. Lao, E. Mendoza, Reaction networks and kinetics of S-systems (submitted)

2. C. Arceo, E. Jose, A. Lao, E. Mendoza, Reaction networks and kinetics of biochemical systems. Math. Biosci. 283, 13-29 (2017)

3. C. Arceo, E. Jose, A. Marin-Sanguino, E. Mendoza, Chemical reaction network approaches to biochemical systems theory. Math. Biosci. 269, 135-152 (2015)

4. B. Boros, On the positive steady states of deficiency one mass action systems. Ph.D. thesis, Eotvos Lorand University (2013)

5. G. Craciun, M. Feinberg, Multiple equilibria in complex chemical reaction networks: the injectivity property. SIAM J. Appl. Math. 65(5), 1526-1546 (2005)

6. M. Feinberg, Lectures on chemical reaction networks (1979). University of Wisconsin. http://www. crnt.osu.edu/LecturesOnReactionNetworks

7. M. Feinberg, F. Horn, Chemical mechanism structure and the coincidence of the stoichiometric and kinetic subspaces. Arch. Ration. Mech. Anal. 66, 83-97 (1977)

8. E. Feliu, C. Wiuf, A computational method to preclude multistationarity in networks of interacting species. Bioinformatics 29(18), 2327-2334 (2013)

9. N. Fortun, A. Lao, L.F. Razon, E. Mendoza, A deficienvy zero theorem for a class of power law kinetic systems with independent decompositions (in preparation)

10. J. Gunawardena, Lectures on chemical reaction network theory for in-silico biologists (2003). Lecture Notes at Bauer Center Harvard

11. M. Johnston, D. Siegel, Linear conjugacy of chemical reaction networks. J. Math. Chem 49, 1263-1282 (2011)

12. S. Müller, G. Regensburger, Generalized mass action systems: complex balancing equilibria and sign vectors of the stoichiometric and kinetic order subspaces. SIAM J. Appl. Math. 72(6), 1926-1947 (2012)

13. S. Müller, G. Regensburger, Generalized mass action systems and positive solutions of polynomial equations with real and symbolic exponents. In: Proceedings of the international workshop on computer algebra in scientific computing (CASC 2014), Lecture Notes in Computing Science (2014)

14. I. Otero-Muras, J. Banga, A. Alonso, Characterizing multiregenstationarity regimes in biochemical reaction networks. PLoS ONE 7(7) (2012)

15. G. Shinar, M. Feinberg, Design principles for robust biochemical reaction networks: What works, what cannot work and what might almost work. Math. Biosci. 231, 39-48 (2011)

16. D. Talabis, Positive equilibria of a class of power law kinetics (2016). MS Thesis, UP Diliman

17. M. Thomson, J. Gunawardena, The rational parametrization theorem. J. Theor. Biol. 261(4), 626-636 (2009) 